\title{
Real versus Artificial Variation in the Thermal Sensitivity of Biological Traits
}

\author{
Samraat Pawar, ${ }^{1, *}$ Anthony I. Dell, ${ }^{2}$ Van M. Savage, ${ }^{3,4,5}$ and Jennifer L. Knies ${ }^{6}$ \\ 1. Department of Life Sciences, Imperial College London, Silwood Park Campus, Buckhurst Road, Ascot, Berkshire SL5 7PY, United Kingdom; \\ 2. National Great Rivers Research and Education Center (NGRREC), East Alton, Illinois 62024; 3. Department of Ecology and Evolutionary \\ Biology, University of California, Los Angeles, California 90095; 4. Department of Biomathematics, David Geffen School of Medicine, \\ University of California, Los Angeles, California 90095; 5. Santa Fe Institute, Santa Fe, New Mexico 87501; 6. Department of Molecular Biology \\ and Chemistry, Christopher Newport University, Newport News, Virginia 23606
}

Submitted November 22, 2014; Accepted July 23, 2015; Electronically published January 5, 2016

Online enhancements: appendixes.

\begin{abstract}
Whether the thermal sensitivity of an organism's traits follows the simple Boltzmann-Arrhenius model remains a contentious issue that centers around consideration of its operational temperature range and whether the sensitivity corresponds to one or a few underlying rate-limiting enzymes. Resolving this issue is crucial, because mechanistic models for temperature dependence of traits are required to predict the biological effects of climate change. Here, by combining theory with data on 1,085 thermal responses from a wide range of traits and organisms, we show that substantial variation in thermal sensitivity (activation energy) estimates can arise simply because of variation in the range of measured temperatures. Furthermore, when thermal responses deviate systematically from the Boltzmann-Arrhenius model, variation in measured temperature ranges across studies can bias estimated activation energy distributions toward higher mean, median, variance, and skewness. Remarkably, this bias alone can yield activation energies that encompass the range expected from biochemical reactions (from $\sim 0.2$ to $1.2 \mathrm{eV}$ ), making it difficult to establish whether a single activation energy appropriately captures thermal sensitivity. We provide guidelines and a simple equation for partially correcting for such artifacts. Our results have important implications for understanding the mechanistic basis of thermal responses of biological traits and for accurately modeling effects of variation in thermal sensitivity on responses of individuals, populations, and ecological communities to changing climatic temperatures.
\end{abstract}

Keywords: temperature, thermal sensitivity, activation energy, sampling, traits, thermal physiology.

\section{Introduction}

Mechanistic mathematical models for the temperature dependence of biological traits that are soundly based on physiological principles will greatly improve forecasts for how populations and ecological communities will respond

* Corresponding author; e-mail: s.pawar@imperial.ac.uk.

Am. Nat. 2015. Vol. 187, pp. E000-E000. (C) 2015 by The University of Chicago. 0003-0147/2016/18702-55909\$15.00. All rights reserved. DOI: $10.1086 / 684590$ to climate change (Deutsch et al. 2008; Angilletta 2009; Dell et al. 2011; Kingsolver et al. 2011; Huey et al. 2012; Pawar et al. 2015). Thermal responses of biological traits are typically unimodal when measured over a sufficiently wide range of temperatures, with performance peaking at some value $T_{\mathrm{pk}}$, beyond which it decreases as deleterious effects of high temperature set in (fig. 1). However, organisms typically operate at ambient temperatures below $T_{\mathrm{pk}}$, within their "physiological temperature range" (PTR; fig. 1; Savage et al. 2004; Deutsch et al. 2008; Martin and Huey 2008; Huey and Kingsolver 2011). Therefore, both thermal sensitivity within the PTR and location of the $T_{\mathrm{pk}}$ are particularly important, because the $0.3^{\circ}-4.8^{\circ} \mathrm{C}$ increase in environmental temperatures projected to take place by the end of this century (Stocker et al. 2013) will impact many organisms by shifting temperatures up, although they will still typically be within the PTR (but see Sunday et al. 2014). Therefore, thermal sensitivity (or "activation energy," as defined below) of traits is likely to have strong effects on the responses of biological systems to climate change (Deutsch et al. 2008; Dillon et al. 2010; Seebacher et al. 2014; Pawar et al. 2015). Even without climate change, Earth has a complex thermal landscape that fluctuates in space and time, and the thermal sensitivity of organisms likely plays a key role in determining biodiversity dynamics (Allen et al. 2002; Kearney and Porter 2009).

The Boltzmann-Arrhenius (BA) model of biochemical kinetics has been proposed as a general mechanistic model for predicting the thermal response of a trait within the PTR (Kooijman 2000; Gillooly et al. 2001; Brown et al. 2004; Savage et al. 2004). For a biological trait (B), the BA model is

$$
B=B_{0} e^{-E / k T},
$$

where $B_{0}$ is a temperature-independent coefficient that includes the effect of body size, $T$ is body temperature in degrees 

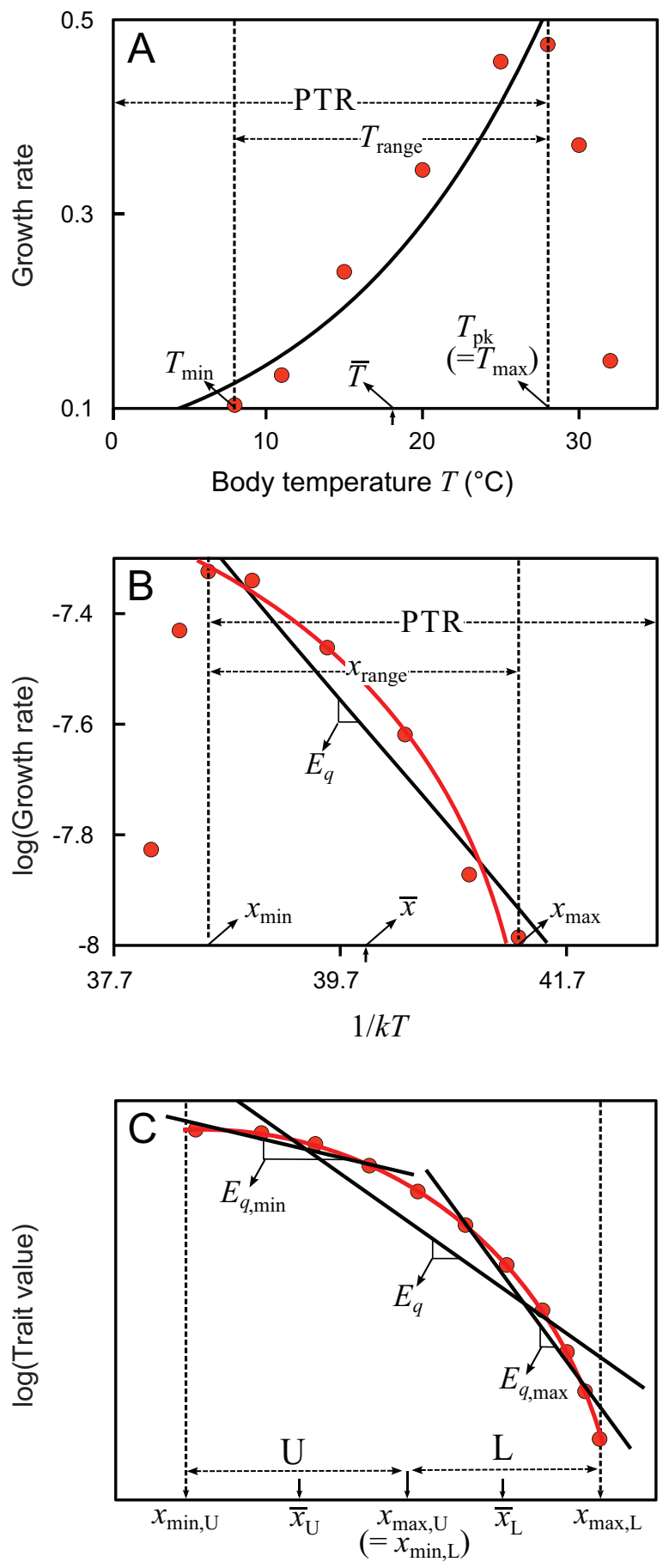

$1 / k T$

Figure 1: $A$, Thermal response curve for radial growth rate $\left(\times 10^{-7} \mathrm{~m} \mathrm{~s}^{-1}\right)$ of a fungal (Metarhizium species) colony (Ouedraogo et al. 1997, included in the Biotraits database; Dell et al. 2013), with the BoltzmannArrhenius (BA) model fitted within the physiological temperature range (PTR). B, Arrhenius plot for the same data with both straight line (black line) and quadratic (red line) models fitted to the data using ordinary least squares (OLS). We define the PTR to be from the lowest theoret-
Kelvin $(\mathrm{K}), k$ is Boltzmann's constant $\left(8.617 \times 10^{-5} \mathrm{eV} \mathrm{K}^{-1}\right)$, and $E$ is the average activation energy in electron volts $(\mathrm{eV})$ of rate-limiting metabolic reactions underlying the trait (Johnson et al. 1974; Gillooly et al. 2001; Brown et al. 2004; Ratkowsky et al. 2005). Thus, $E$ determines the rate of exponential response (thermal sensitivity) of the trait within the PTR. Note that, under the dynamic energy budget (DEB) theory (Kooijman 2000; Freitas et al. 2010), an equivalent formulation to equation (1) is used to capture the thermal dependence of physiological traits, with the focus instead being on the "Arrhenius temperature," $T_{\mathrm{a}}=E / k$; that is, the equation used is $B=B_{0} \exp \left(-T_{a} / T\right)$. This may be interpreted as a greater agnosticism of the DEB toward whether $E$ indeed reflects the activation energy of one or more ratelimiting enzymes in the underlying metabolic pathways. Indeed, whether the thermal sensitivities of biological traits truly reflect an activation energy remains a contentious issue (Clarke 2004; Gillooly et al. 2006; del Rio 2008; Hobbs et al. 2013; Corkrey et al. 2014). Use of the BA model in ecology has also been criticized, because recent empirical studies have revealed a much wider range of $E$ values (Irlich et al. 2009; Knies and Kingsolver 2010; Dell et al. 2011; Englund et al. 2011) than the $0.6 \mathrm{eV}$ (or sometimes $0.65 \mathrm{eV}$ ) originally suggested (Gillooly et al. 2001) and which has subsequently so often been used in theoretical studies (Savage et al. 2004; Vasseur and McCann 2005; Wolfshaar et al. 2008; Petchey et al. 2010; Rall et al. 2010; O'Connor et al. 2011; Stegen et al. 2012). Understanding the sources of the variation in $E$ within and across species is therefore important, especially because such variation is likely to have nontrivial effects on ecological and evolutionary dynamics (Vasseur and McCann 2005; Dell et al. 2011, 2014).

While some of the variation in $E$ clearly has a biological basis (Johnson et al. 1974; Dell et al. 2011; Corkrey et al. 2012), the choice of experimental temperature range combined with fitting artifacts may play a major role by undermining the accuracy and precision of $E$ estimates. In par-

ical nonlethal temperature at which the trait value is nonzero (here assumed to be the freezing point of water, $0^{\circ} \mathrm{C}$ ) to that of peak performance $\left(T_{\mathrm{pk}}\right)$. Because this experiment was performed over a sufficiently wide temperature range to capture $T_{\mathrm{pk}}$, here $T_{\max }=T_{\mathrm{pk}}$. Data points at temperature above $T_{\max }$ (thick vertical dashed lines in $A$ and $B$ ) are excluded from model fits. Other key parameters, including range, range-location, and range-spread, are defined in the text (eqq. [4][6]). Note that $E_{q}$ denotes the activation energy calculated by forcing a straight line through a curved Arrhenius plot as illustrated here. $C$, Effect of temperature range and range-location on the calculation of $E_{q}$. The long solid black line is a unique straight line that fits the curved data (here shown to cover the entire PTR) using the OLS criterion. $E_{q}$ will be overestimated if experimental temperatures are limited to the lower end of the PTR (steep line through data in range L, corresponding to $\bar{x}_{\mathrm{L}}$ ) and underestimated if temperatures are limited to the upper end of the PTR (shallow line through data in range U, corresponding to $\bar{x}_{U}$ ). 
ticular, Knies and Kingsolver (2010) showed how, if the upper cutoff for the PTR is defined to be exactly $T_{\mathrm{pk}}$, the Arrhenius plot will show curvature, because as trait values approach $T_{\mathrm{pk}}$, they no longer increase exponentially (fig. $1 B$, $1 C)$. And even if the upper cutoff for the PTR is redefined below $T_{\mathrm{pk}}$ to minimize this effect, thermal responses of biological traits can deviate from the strict BA model by being weaker than exponential, partly due to the complexity of the underlying metabolic pathways, such as regulation by multiple rate-limiting enzymes (Wolfe and Bagnall 1980; Silvius and McElhaney 1981; Ratkowsky et al. 2005; Knies and Kingsolver 2010; Dell et al. 2011). For example, in contrast to equation (1), metabolic rates may exhibit different apparent low- and intermediate-temperature activation energies within the PTR separated by an "Arrhenius breakpoint temperature" (Sharpe and DeMichele 1977; Schoolfield et al. 1981; Silvius and McElhaney 1981; Corkrey et al. 2012) within the PTR, which essentially implies a nonlinear Arrhenius plot (fig. 1). Other possible sources of curvature in the Arrhenius may include changes in heat capacity of rate-limiting enzymes (Hobbs et al. 2013) and the combined effect of MichaelisMenten and Arrhenius kinetics (Kruse et al. 2011).

Regardless of the underlying reason, when Arrhenius plots exhibit downward (or upward) curvature, $E$ is bound to vary with location of the experimental temperature range (maximum measured temperature - minimum measured temperature, or $T_{\max }-T_{\min }=T_{\text {range }}$; fig. $1 A$ ). For downward curvature, $E$ will be underestimated if temperatures are chosen at the upper end of the PTR (temperature range $U$ in fig. 1) and overestimated if temperatures are chosen at the lower end of the PTR (temperature range L in fig. 1). Therefore, to properly assess statistical fits to the BA model and to obtain more realistic estimates of $E$ for use in ecological and evolutionary studies on effects of climate change, it is necessary to quantify effects of artifacts on estimates of $E$, such as the choice of range of temperatures included in the analysis, or location of the temperature range (range-location) within the PTR.

In this article, we develop an analytical framework for understanding how fitting artifacts and systematic deviations from the BA model together affects the accuracy and precision of $E$ estimates for the thermal response of biological traits. Henceforth, by "experimental artifacts," we mean the effect on estimated $E$ of choosing an inappropriate experimental temperature range or insufficient replication; by "fitting artifacts," we mean the effect of choosing an experimental temperature range inappropriate for fitting the BA model; and by "systematic deviations," we mean the case where the underlying thermal response, if sampled adequately, deviates systematically from the $\mathrm{BA}$ model such that the Arrhenius plot is curved, even if the effect of $T_{\mathrm{pk}}$ is excluded. In terms of experimental or fitting artifacts, we separate the largely independent effects of choice of experimental (or model fitting) temperature range and range-location (center of the temperature range). We test our framework using 1,085 intraspecific thermal-response curves with ranges from $5^{\circ}$ to $45^{\circ} \mathrm{C}$ for a diversity of physiological and ecological traits, and we consider whether and how the accuracy and precision of $E$ estimates across studies may be significantly affected by artifacts combined with systematic deviations.

\section{Theory}

The key equation for BA temperature dependence (eq. [1]) can be represented in logarithmic space (fig. 1) as

$$
\log (B)=\log \left(B_{0}\right)-E(1 / k T),
$$

(equivalent to $\log (B)=\log \left(B_{0}\right)-T_{\mathrm{a}}(1 / T)$ under $\left.\mathrm{DEB}\right)$. When a trait is measured at $n$ distinct temperatures, this equation can be cast as a linear regression model:

$$
y_{i}=c+m x_{i}+\varepsilon_{i}, \quad i=1, \ldots, n,
$$

where $m \equiv-E, x_{i} \equiv 1 / k T_{i}, y_{i} \equiv \log \left(B_{i}\right), c \equiv \log \left(B_{0}\right)$, and $\varepsilon_{i}$ are uncorrelated random errors assumed to be such that the expectation value or mean random error across all data points is zero (i.e., $E\left(\varepsilon_{i}\right)=0$ ). We can calculate the variance in the random error, which is of course just the square of the standard deviation (i.e., $\operatorname{Var}\left(\varepsilon_{i}\right)=\sigma^{2}$ ). We assume that temperatures $T_{i}$ have minimal errors, because they are controlled by experimentalists. Thus, we assume that $\operatorname{Var}\left(y_{i}\right) \propto \sigma^{2}$; that is, the variance in $y$ over and above the variation due to the trait's thermal response itself arises from the random errors $\varepsilon_{i}$. We note that, even though a precision for temperature higher than $\sim 0.1^{\circ} \mathrm{C}$ is typically not achievable in biological experiments, what matters is that, as a rule of thumb, this precision is at least three times that of the measurements of the $Y$-axis variable (the metabolic trait, in this case; McArdle et al. 1988). Also, we assume that the trait response data are mutually uncorrelated and homoscedastic $\left(E\left[\varepsilon_{i}^{2} \mid Y\right]=\sigma^{2}\right)$. We henceforth deal mainly with $\log$ of metabolic rate $(\log (B))$ and reciprocal temperature $(1 / k T)$, because this is the transformed measurement scale in which coefficients of the BA model are estimated (eqq. [2] and [3]; fig. 1). We use ordinary least squares (OLS) regression analysis to estimate coefficients $\log \left(B_{0}\right)$ and $E$. Figure $1 B$, known as an Arrhenius plot, illustrates the transformation from equation (1) to (2) and the OLS fit of the model described by equation (3) to a real data set.

We consider both accuracy (how close the estimated value is to its real value) and precision (how much the estimate varies due to random errors) in the estimation of $E$. To this end, for each thermal response, we define $T_{\min }$ as the minimum temperature (Kelvin) at which the trait was measured 
and $T_{\max }$ as the temperature at which the maximum trait value was recorded within the PTR (fig. $1 A$ ). Note that, in some previous studies, $T_{\min }$ has been defined to be the theoretical minimum temperature at which trait values are still measurable (i.e., the lower limit of the PTR in fig. $1 A$; e.g., McMeekin et al. 2013), not the minimum temperature at which a trait value was actually measured, as we define it here. Similarly, it should also be noted that some previous studies have used $T_{\max }$ to denote the highest theoretical maximum temperature beyond the upper limit of the PTR for a nonzero trait value (McMeekin et al. 2013). Within our definition, only when an organism's PTR is fully covered in the experiment is $T_{\max }=T_{\mathrm{pk}}$ (fig. 1).

Thus, $x_{\min }=1 / k T_{\max }$ and $x_{\max }=1 / k T_{\min }$ (units of $\mathrm{eV}^{-1}$; fig. $1 B$ ). Then, within the PTR, we can define the following key measures. First, the reciprocal temperature range is

$$
x_{\text {range }} \equiv \frac{1}{k T_{\min }}-\frac{1}{k T_{\max }} \propto T_{\text {range }}\left(=T_{\max }-T_{\min }\right) .
$$

That is, $x_{\text {range }}$ is proportional to $T_{\text {range }}$ Second, the reciprocal temperature range-location is

$$
\bar{x} \equiv \frac{1}{n} \sum_{i=1}^{n} \frac{1}{k T_{i}} \approx \frac{1}{k \bar{T}},
$$

where $\bar{T}$ is the mean of the experimental temperatures and $\bar{x} \propto 1 / \bar{T})$. Third, the reciprocal temperature range-spread is

$$
x_{\text {spread }} \equiv \sum_{i=1}^{n}\left(x_{i}-\bar{x}\right)^{2} \propto T_{\text {spread }}\left(=\sum_{i=1}^{n}\left(T_{i}-\bar{T}\right)^{2}\right) .
$$

That is, $x_{\text {spread }}$ is approximately proportional to $T_{\text {spread }}$. Note that $x_{\text {spread }}$ is also proportional to $x_{\text {range }}$ and therefore proportional to $T_{\text {range }}$ (eq. [4]; fig. 1).

Thus, we have measures of three key sources of variation in experimental design: temperature range $x_{\text {range }}\left(\propto T_{\text {range}}\right.$; eq. [4]), which measures how much of width of the PTR an experiment has covered; range-spread $x_{\text {spread }}\left(\propto \sum_{i=1}^{n}\right.$ $\left(T_{i}-\bar{T}\right)^{2}$; eq. [6]), which measures how uniformly $T_{\text {range }}$ has been sampled; and range-location $\bar{x}(\approx 1 / k \bar{T}$; eq. [5]), which measures where $T_{\text {range }}$ is located. The approximation in equation (5) and the proportionalities in equations (4) and (6) are all valid in the region of the PTR, even though $x(=1 / k T)$ is, overall, a hyperbolically declining function of $T$. This is because $1 / k T$ is approximately linear over the temperature range covered by the PTR, which averages $\sim 25^{\circ} \mathrm{C}$ in well-sampled thermal responses (Ratkowsky et al. 2005; Knies and Kingsolver 2010; Dell et al. 2011; app. B; apps. A-C available online). Thus, conclusions drawn in terms of $x$ can be directly interpreted in terms of the range, range-spread, and range-location of $T$. Also, in real experimental data, $T_{\text {range }}$ often does not cover the full PTR. Indeed, one of our main aims is to quantify the biases introduced in estimates of $E$ due to an incomplete coverage of the PTR.

\section{Estimating E When the Arrhenius Plot Is Not Curved}

We first consider the accuracy and precision of $\hat{E}$ when the trait thermal response shows no curvature on an Arrhenius plot (unlike the scenarios shown in fig. 1). As detailed later, this curvature can be detected by fitting a quadratic regression model to the Arrhenius plot. Then, for the OLS regression model for the Arrhenius plot (eq. [3]) without curvature, the OLS estimator of $m$ is (Lehmann and Casella 1998; app. A):

$$
\hat{m}=-\hat{E}=\frac{\sum_{i=1}^{n}\left(x_{i}-\bar{x}\right)\left(y_{i}-\bar{y}\right)}{\sum_{i=1}^{n}\left(x_{i}-\bar{x}\right)^{2}}=\frac{\operatorname{Cov}(x, y)}{\operatorname{Var}(x)} .
$$

Thus, the accuracy of $\hat{E}$ is independent of reciprocal temperature range ( $x_{\text {range; }}$ eq. [4]), range-location $(\bar{x}$; eq. [5]), and range-spread ( $x_{\text {spread }}$; eq. [6]). Provided that errors $\varepsilon_{i}$ (eq. [3]) are uncorrelated and homoscedastic (Lehmann and Casella 1998), it can be shown that equation (7) is also the best linear unbiased estimator (most accurate estimator that is unbiased) of $E$. That is, the average $\hat{E}$ from a sufficiently large number of replicated experiments will converge on the true value of $E$ (see fig. $2 A$ ), irrespective of $x_{\text {range }}, \bar{x}$, and $x_{\text {spread }}$.

Now we consider the precision of $\hat{E}$. Given that $\operatorname{Var}(\hat{E})=\sigma^{2} / x_{\text {spread }}$ and that an unbiased estimator of $\sigma^{2}$ is $s^{2}=\sum_{i=1}^{n} \hat{\varepsilon}_{i}^{2} /(n-2)$, where $\hat{\varepsilon}_{i}=y_{i}-c+\hat{E} x_{i}$ (the regression residuals), the standard error of $\hat{E}$, which we will designate by $s_{\hat{E}}$, is as follows (Lehmann and Casella 1998):

$$
s_{\hat{E}}=\sqrt{\frac{\sum_{i=1}^{n} \hat{\varepsilon}_{i}^{2}}{x_{\text {spread }}(n-2)}}=\sqrt{\frac{\sum_{i=1}^{n}\left(y_{i}-\hat{c}+\hat{E} x_{i}\right)^{2}}{x_{\text {spread }}(n-2)}} .
$$

That is, precision increases ( $s_{\hat{E}}$ decreases) with temperature range-spread $x_{\text {spread }}$ (eq. [6]). For a given level of trait measurement error $\varepsilon$ and $n$ experimental temperatures, a straightforward method for the experimentalist to increase $x_{\text {spread }}$ is to widen $T_{\text {range }}$ within the PTR. Of course, if $T_{\text {range }}$ and $n$ of an experiment are fixed, $x_{\text {spread }}$ (and therefore precision) can be maximized within these constraints by dividing the $n$ measurements equally between the ends of the $T_{\text {range }}$. However, without sampling points between the end points of the $T_{\text {range, }}$ it would be impossible to detect curvature in 

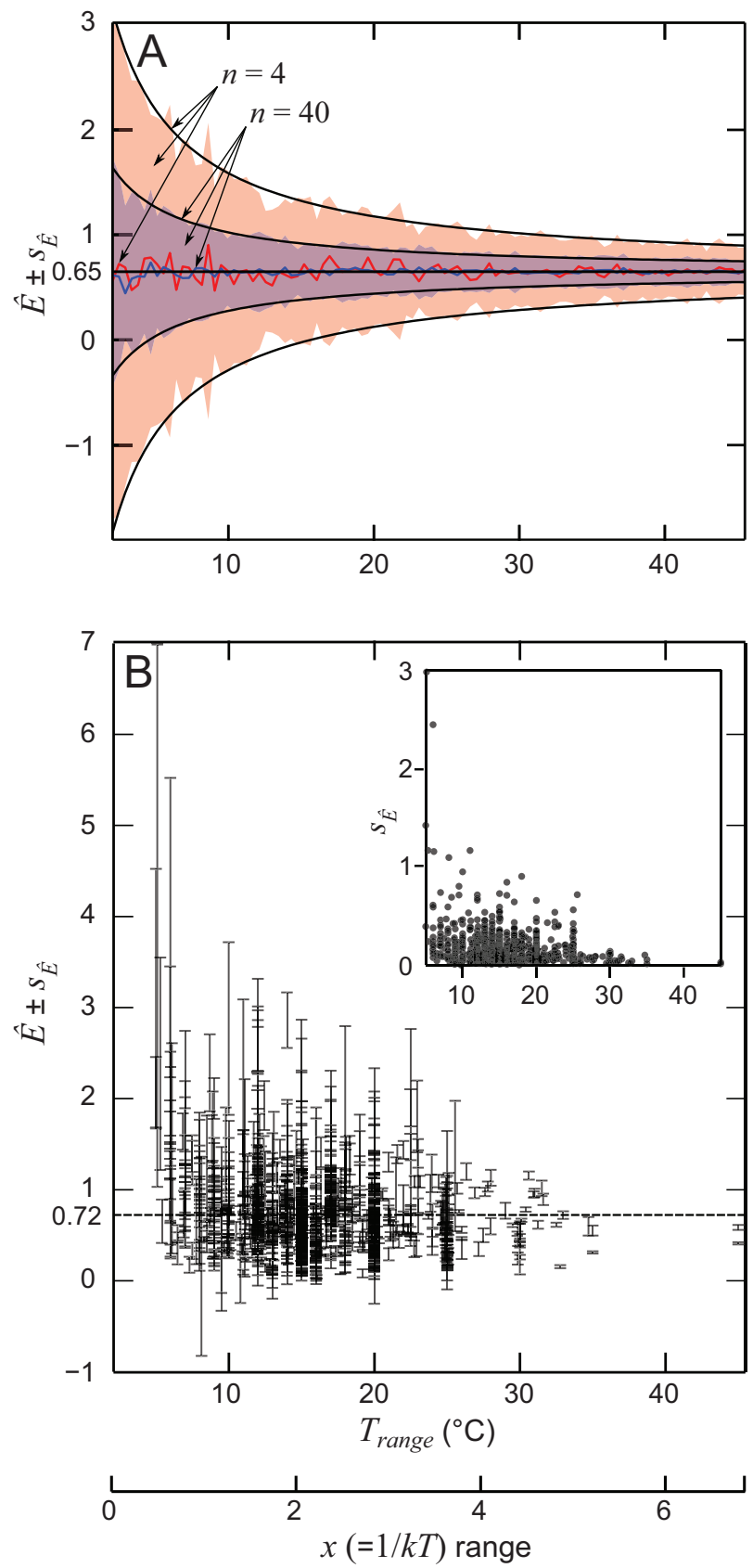

Figure 2: Effect of experimental temperature range ( $T_{\text {range; }}$; eq. [4]) on the accuracy and precision of activation energies $(\hat{E})$ of thermal responses, estimated from ordinary least squares (OLS) fits in the Arrhenius plot. We use $T_{\text {range }}$ instead of range-spread ( $T_{\text {spread }}$ eq. [6]) here because $T_{\text {range }}$ strongly determines $T_{\text {spread }}$ and is a directly interpretable quantity. $A$, Simulation results (colored lines or shaded regions) overlaid on the analytical solutions (solid black lines) for accuracy (eq. [7]) and precision (eq. [8]) of $\hat{E}$ for two different levels of sampling of temperature points $(n)$ within a range. In the simulations, $\hat{E}$ at a given $T_{\text {range }}$ was obtained by generating 100 thermal responses (equivalent to experimental replicates) for a perfect Boltzmann-Arrhenius (BA) response of $E=0.65 \mathrm{eV}$, with Gaussian errors of mean 0 and variance 1 (in accordance with eqq. [3] and [9]). The sample mean and standard devi- the Arrhenius plot (see below) and, therefore, systematic deviations from the BA model.

These analytic results for the dependence of accuracy and precision of $\hat{E}$ (eqq. [7], [8]) on experimental temperature range and range-location are illustrated using simulations in figure $2 A$. Note that, at the lowest $x_{\text {range, }}$ an order of magnitude increase in $n$, from 4 to 40 , can double precision. Conversely, for the minimum feasible $n$ temperature samples $(n=4)$, an order of magnitude increase in range can triple precision (fig. $2 A$ ). Thus, the greatest benefit for maximizing precision of $\hat{E}$ will come from increasing the temperature range.

\section{Estimating E When the Arrhenius Plot Is Curved}

We now consider the case where the thermal response of a trait deviates from the BA model such that the Arrhenius plot has a concave downward curvature, which we term "BAlike" (fig. $3 B$ ). The simplest regression model that allows for curvature is a quadratic model (Knies and Kingsolver 2010; Dell et al. 2011; Englund et al. 2011), so we use that as an easy way to modify our linear-regression equation and to obtain some measure of curvature in the Arrhenius plot:

$$
y_{i}=q_{0}+q_{1} x_{i}+q_{2} x_{i}^{2}+\varepsilon_{i}, i=1, \ldots, n,
$$

where $y_{i}$ is the trait value (same as in eq. [3]), $q_{0}, q_{1}$, and $q_{2}$ are fitted coefficients, and $\varepsilon_{i}$ values are uncorrelated errors as specified for the linear model (eq. [3]). The coefficient $q_{2}$ will be negative, because the quadratic curvature is downward. Even for such data, where a quadratic model (eq. [9]) would be more appropriate, we can still estimate an "activation energy" for the thermal response using the usual OLS estimator (eq. [7]) by forcing a straight line model (eq. [3]) through the Arrhenius plot where the slope $m=-E_{q}$, with the sub-

ation (i.e., standard error $s_{\hat{E}}$ ) from the $100 \hat{E}$ values were then calculated. The maximum possible $T_{\text {range }}$ was set to be $45^{\circ} \mathrm{C}\left(T_{\min }=0^{\circ} \mathrm{C}\right.$, $T_{\max }=45^{\circ} \mathrm{C}$ ), because $45^{\circ} \mathrm{C}$ is approximately the highest $T_{\text {max }}$ observed in the data. The minimum possible $T_{\text {range }}$ was set to be $1^{\circ} \mathrm{C}$ $\left(x_{\text {range }} \approx 0.15 \mathrm{eV}^{-1}\right)$. A lower limit of $n=4$ was chosen because OLS regression analyses require $n-2$ degrees of freedom, which also happens to be close to the median $n$ in the analyzed data set. Provided range-location is centered within the physiological temperature range (PTR), the theoretical results for dependence of accuracy and precision on range-location are identical for BA-like responses with curved Arrhenius plots (see main text). $B$, Dependence of accuracy and precision of $\hat{E}$ for individual empirical thermal responses (experiments). The greater variation in mean and standard error in $\hat{E}$ values from the experimental data, compared with the simulated data in $A$, arises because each $\hat{E}$ in the empirical data may be from a different population or trait, thus introducing additional sources of variation. The inset shows centered $s_{\hat{E}}$ values from the estimate from individual experiments. The dashed line marks the mean $\hat{E}$. 

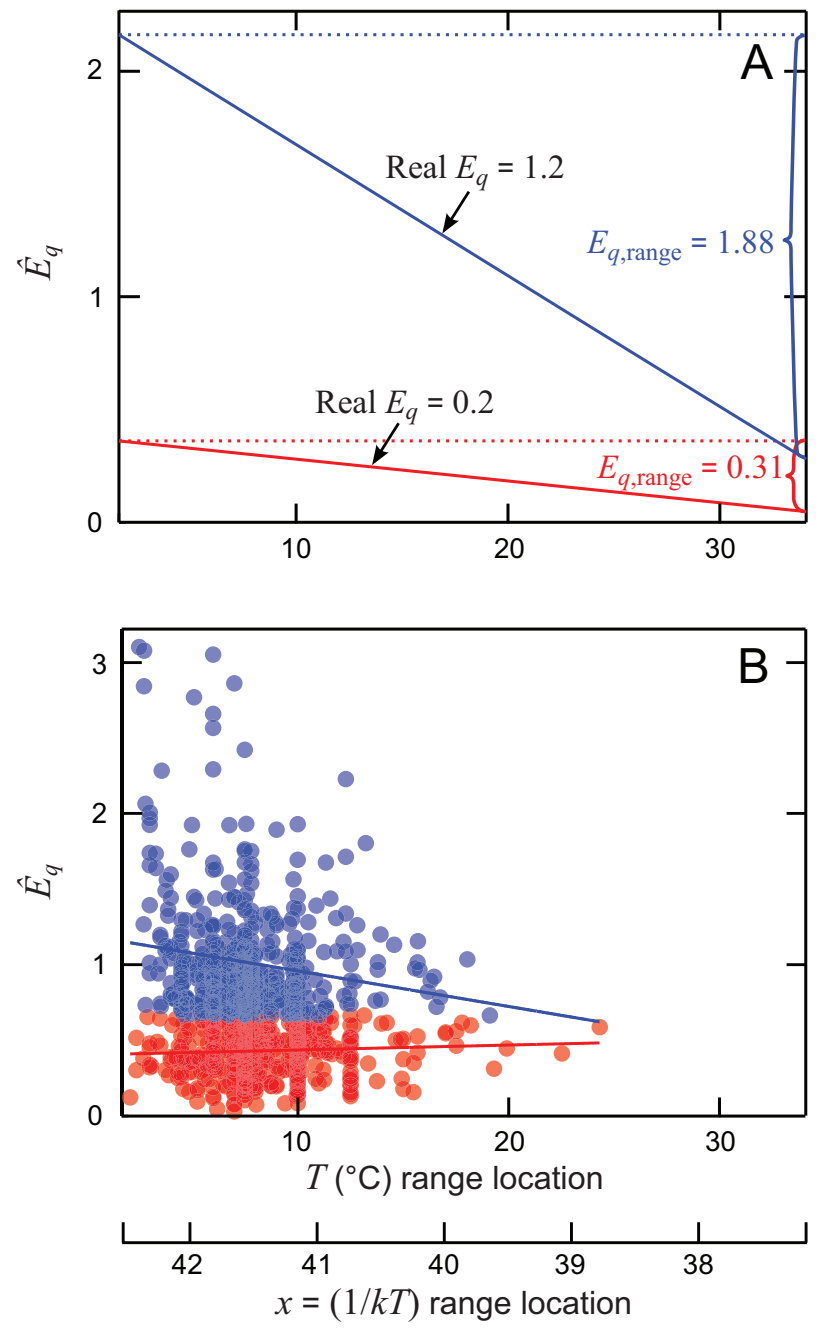

Figure 3: Effect of experimental temperature range-location (eq. [5]) in Boltzmann-Arrhenius-like thermal responses (with curved Arrhenius plots) on ranges of activation energy estimates, $\hat{E}_{q}$. A, Theoretically predicted $\hat{E}_{q, \text { range }}$ values arising from the linear relationship between $\hat{E}_{q}$ and range-location $\bar{x}\left(\hat{E}_{q} \approx q_{1}-2 q_{2} \bar{x}\right.$; eq. [10]) shown by the two sloping solid lines. The two lines are for the two extreme values of activation energies $\left(E_{q}=0.2\right.$ and $\left.E_{q}=1.2\right)$ expected from the metabolic theory of ecology and illustrate how much $\hat{E}_{q}$ may vary (magnitude of $\hat{E}_{q, \text { range }}$ ) if the Arrhenius plot showed curvature. They also show how the range encompassed by any one line $\left(\hat{E}_{q, \text { range }}\right)$ increases with the magnitude of the real $\hat{E}_{q}$ (eq. [11]). Note that the quadratic curvature, determined by the coefficients $q_{1}, q_{2}$, necessarily varies proportionally with $\hat{E}_{q}$, as discussed in the main text following equation (10). $B$, The effect of range-location on $\hat{E}_{q}$ in the empirical data, calculated for a subset of the thermal responses that have negative curvature. The solid blue and red lines are fitted ordinary least squares (OLS) regressions to sets of activation energies above and below the median value, respectively. As in $A$, higher values of $\hat{E}_{q}$ (above-median values, blue markers) show a greater sensitivity to range location (bigger $\hat{E}_{q, \text { range }}$ ) than the lower values (red markers). Consistent with this, as expected from equation (10), the above-median values of $\hat{E}_{q}$ correspond to higher curvature coefficients (mean $\hat{q}_{2}=-0.32$, mean $\hat{q}_{1}=24.2$ ) than the belowmedian values of $\hat{E}_{q}$ (mean $\hat{q}_{2}=-0.022$, mean $\hat{q}_{1}=1.56$ ). script $q$ denoting that this is not an activation energy in the strict sense.

As illustrated in figure $1 C$, however, the accuracy of $E_{q}$ will be sensitive to which portion of the quadratic curve (and thus PTR) the straight line is forced through: $\hat{E}_{q}$ will be an underestimate if experimental temperatures are chosen at the upper end of the PTR (high $\bar{T}$, low $\bar{x}$ ) and an overestimate if temperatures are chosen at the lower end of the PTR (low $\bar{T}$, high $\bar{x}$; fig. 1 ). Thus, $\hat{E}_{q}$ will vary with the choice of experimental $T_{\text {range, }} T_{\text {spread }}$ and $\bar{T}$. To derive this dependence mathematically, we ignore measurement errors (i.e., we assume the Arrhenius plot of the data perfectly follows the quadratic model) and substitute equation (9) into equation (1), which simplifies to the approximation (see app. A)

$$
\hat{m}=-\hat{E}_{q} \approx q_{1}+2 q_{2} \bar{x} \approx q_{1}+2 q_{2}(1 / k \bar{T}),
$$

where, by definition, $q_{2}$ is negative due to the downward curvature. Thus, for curved Arrhenius plots, $\hat{E}_{q}$ depends linearly on the curvature coefficient $q_{2}$ and range-location $\bar{T}$ (eq. [5]), as illustrated in figure $3 A$. Conversely, because coefficients of polynomials are intercorrelated, higher values of $\hat{E}_{q}$ also correspond to higher values of both $q_{1}$ and $q_{2}$. For example, for $\hat{E}_{q}$ in the range $[0.2,1.2]$, as expected from the metabolic theory of ecology (Gillooly et al. 2001), $q_{1}$ lies in the range $[2.85,17.13]$, and $q_{2}$ lies in the range $[-0.04,-0.23]$. Of course, as the curvature vanishes $\left(q_{2} \rightarrow 0\right)$, then $q_{1} \rightarrow \hat{E}$ (i.e., $\hat{E}_{q} \rightarrow \hat{E}$ ). The approximation in equation (10) is valid as long as the experimental temperatures $T_{i}$ are spaced somewhat evenly, such as in an arithmetic series. Extensive data on thermal response experiments show that this is a valid assumption (app. A), reflecting the fact that experimentalists typically select evenly spaced temperature treatments.

We next consider how the precision of $\hat{E}_{q}$ depends on $T_{\text {range, }} T_{\text {spread, }}$ and $\bar{T}$. Analogous to equation (8), the standard error of $\hat{E}_{q}$ can be estimated from deviations $\left(\varepsilon_{i}\right)$ around the linear model (eq. [3]) forced through it. Although the errors are actually around the underlying quadratic curve (eq. [9]), by definition, the OLS regression line through a quadratic curve with errors still minimizes the sum of the net deviations. Therefore, equation (8) is also appropriate for measuring the precision of $\hat{E}_{q}$ from BA-like responses. That is, as in the case of $\hat{E}$, the precision of $\hat{E}_{q}$ too will decrease with $T_{\text {spread }}$ (eq. [8]), and therefore $T_{\text {range, }}$, as illustrated in figure $2 A$.

Furthermore, we can measure the sensitivity of $\hat{E}_{q}$ to the amount of curvature (curvature sensitivity) exhibited by the thermal response of the trait on an Arrhenius plot. As such, curvature sensitivity of the $E_{q}$ is embodied in the coefficient $q_{2}$. We can translate this coefficient into the range of activa- 
tion energies that any particular BA-like thermal response can generate, as follows. Let us split the experimental temperature range $\left(T_{\max }-T_{\min }\right)$ into equal lower (L) and upper (U) subranges (fig. 1C). In many cases, instead of a midpoint, an inflection point in the Arrhenius plot may provide a more natural temperature for subdividing the temperature range (indicating an Arrhenius breakpoint temperature; Schoolfield et al. 1981; Silvius and McElhaney 1981). The activation energies of the two temperature subranges (using eq. [10]) are as follows:

$$
\begin{aligned}
& \hat{E}_{q, \text { min }} \approx-\left(q_{1}+2 q_{2} \bar{x}_{U}\right), \\
& \hat{E}_{q, \max } \approx-\left(q_{1}+2 q_{2} \bar{x}_{L}\right) .
\end{aligned}
$$

Then, a measure of curvature sensitivity of $\hat{E}_{q}$ is the difference between these two activation energies:

$$
\begin{aligned}
\hat{E}_{q, \text { range }} \approx \hat{E}_{q, \text { max }}-\hat{E}_{q, \text { min }} & =\left(q_{1}+2 q_{2} \bar{x}_{U}\right)-\left(q_{1}+2 q_{2} \bar{x}_{L}\right) \\
& =2 q_{2}\left(\bar{x}_{U}-\bar{x}_{L}\right) .
\end{aligned}
$$

Thus, for quadratically curved Arrhenius plots, choice of experimental temperatures that yield $\bar{x}$ (and therefore $\bar{T}$ ) close to the center of the PTR will minimize curvature sensitivity of $\hat{E}_{q}$. Note that we call these upper and lower bounds on $\hat{E}_{q}$ "reasonable," because the upper and lower ranges need not necessarily be delineated by splitting the temperature range into exactly two halves.

These analytic results for the dependence of accuracy and precision of $\hat{E}$ and $\hat{E}_{q}$ (eqq. [7], [8], [10], [11]) on experimental $T_{\text {range, }} T_{\text {spread }}$, and $\bar{T}$, with or without curvature of the Arrhenius plot, are validated and illustrated in figures 2 and 3 and juxtaposed against empirical data (see below).

\section{Empirical Results}

Based on our analytical results, we now evaluate sensitivity of $E$ to variation in temperature range-location and rangespread in real data. For this, we combined BioTraits, the largest current database on the thermal responses of biological traits, containing 2,352 responses for 220 traits across microbes, plants, and animals (Dell et al. 2011, 2013) with 35 thermal responses of bacterial growth rates (Ratkowsky et al. 2005) and 283 responses for phytoplankton growth rates (Thomas et al. 2012). All traits were transformed to consistent SI units, as described previously (Dell et al. 2011, 2013). The responses in the combined data set that included a $T_{\mathrm{pk}}$ (unimodal) were curtailed to isolate data only from the PTR (fig. 1). We considered any thermal response as unimodal if it had trait measurements at a minimum of two different temperatures both above and below a potential $T_{\mathrm{pk}}$.

Of the data thus modified to isolate only PTRs, we analyzed all responses that contained nonzero measurements at a minimum of four distinct temperatures and covered a range of at least $5^{\circ} \mathrm{C}$ within the PTR. This yielded 1,085 responses. In these responses, the temperature range for the PTR varies nine-fold, from $5^{\circ} \mathrm{C}\left(x=41.72 \mathrm{eV}^{-1}\right)$ to $45^{\circ} \mathrm{C}\left(x=36.48 \mathrm{eV}^{-1}\right)$. After converting each response to an Arrhenius plot (eq. [2]; fig. 1), we used OLS regression (eq. [3]) to estimate $E$ and its standard error. The resulting distribution of $E$ estimates is right-skewed, as previously reported by Dell et al. (2011), with a mean and median value of $0.72 \mathrm{eV}$ and $0.66 \mathrm{eV}$, respectively. These estimates vary over a hundred-fold (0.027-4.01 eV), emphasizing that the fixed value of 0.6 or $0.65 \mathrm{eV}$ so often used to model thermal sensitivity in climate change-related studies is difficult to justify. Below, we show that the right-skewness of the distribution can partly be explained by curvature in the Arrhenius plot, which causes $\hat{E}$ to decrease with range location $\bar{x}$ (fig. 3).

We next fitted a quadratic curve (eq. [9]) to the Arrhenius plot of each response. Note that we had already curtailed all thermal responses to just the PTR or its measured subset (fig. 1). The quadratic coefficient $\left(q_{2}\right)$ was found to be negative (indicating downward curvature) for 590 (54.4\%) of the thermal responses and positive (indicating zero or upward curvature) for the remaining 45.6\%. Among the 590 thermal responses with downward curvature and enough data points (five) to test for a significantly better fit of a quadratic model as compared to a linear one by smallsample Akaike information criterion (AIC), 193 (32.7\%) are significantly downward curved (i.e., overall, $17.8 \%$ are significantly downward curved). Note that our ability to detect significant negative downward curvature is limited by the fact that most experiments do not cover a sufficient temperature range to cover the entire PTR (i.e., they do not capture $T_{\mathrm{pk}}$, resulting in $T_{\max } \ll T_{\mathrm{pk}}$ ). This decreases the possibility of curvature in the Arrhenius plot arising due solely to effects of approaching $T_{\mathrm{pk}}$. Also, the low sample size of individual responses (median value of $n=6$ for the data sets with downward curvature) limits our ability to detect significant curvature using the small-sample AIC values. Nevertheless, even if not significant on the basis of smallsample AIC values, weak negative curvature is sufficient to generate significant effects on the accuracy of estimated values of $E$ in thermal response data, as indicated by our theory and verified by the following empirical results.

Having separated the data with downward-curved Arrhenius plots (BA-like responses) from the rest, we first test whether, as predicted for the BA model, $\hat{E}$ is independent of temperature range-spread (eq. [7]; fig. 2A), which should also hold for BA-like responses provided that the 
range-location is centered on the PTR. Indeed, we find $E$ is only weakly dependent on temperature range (Spearman's rank correlation, $\left.\mathrm{r}_{\mathrm{s}}=-0.07 ; P=.021\right)$. Next, we examine whether the precision of $\hat{E}$ decreases hyperbolically with temperature range for both $\mathrm{BA}$ and $\mathrm{BA}$-like responses, as predicted (fig. $2 A$ ). Indeed, the precision of $\hat{E}$, the standard $s_{\hat{E}}$, decreases hyperbolically with increasing temperature range (fig. $2 B$ and inset). Similar results are obtained for phytoplankton growth rate, bacterial growth rate, and body velocity, the three largest homogeneous subsets of responses in our data set (app. C).

Third, we distinguish between the fit of the BA and BAlike models to the empirical data. We ask whether, as predicted for the BA model, $\hat{E}$ is independent of range-location $\bar{x}$, as in equation (7) (= inverse of $\bar{T}$; eq. [5]), or whether, as predicted for the BA-like model, for thermal responses with negatively curved Arrhenius plots, the absolute value of $\hat{E}$ decreases linearly with $\bar{T}$ (fig. $3 A$; cf. eq. [10]) — that is, the $\hat{E}$ values are actually $\hat{E}_{q}$ values. Because $T_{\min }$ varies across thermal responses (and therefore range location varies), the temperature range of each thermal response was shifted so that $T_{\min }=0$ to reduce the confounding effect of variation in $T_{\min }$. In contrast to the BA model and consistent with the BA-like prediction, $\hat{E}$ is not independent of range-location, but instead decreases with $\bar{T}$ (Spearman rank-order correlation for $\hat{E}$ vs. $\left.\bar{T}: r_{\mathrm{s}}=0.185, P<.0001\right)$. Furthermore, to gauge whether, as predicted by equation (11), curvature sensitivity $\left(\hat{E}_{q, \text { range }}\right)$ increases with $\hat{E}_{q}$ itself, we divided the $E_{q}$ estimates into two categories: those with values less than and those with values greater than the median value of $\hat{E}_{q}$ (fig. $3 B$ ). As expected, a much larger $\hat{E}_{q \text {,range }}(0.66-3.10$ $\left.\mathrm{eV} ; \hat{E}_{q, \text { range }}=2.44 \mathrm{eV}\right)$, is observed for the larger values of $\hat{E}_{q}$ than for the smaller values $\left(0.027-0.66 \mathrm{eV} ; \hat{E}_{\text {q,range }}=0.64\right.$ $\mathrm{eV})$. Consistent with this, as expected from equation (10), the above-median values of $\hat{E}_{q}$ correspond to higher curvature coefficients (mean $\hat{q}_{2}=-0.32$, mean $\hat{q}_{1}=24.2$ ) than the below median values of $\hat{E}_{q}$ (mean $\hat{q}_{2}=-0.022$, mean $\left.\hat{q}_{1}=1.56\right)$ and the correlation of $\hat{E}_{q}$ with range-location $T$ is significantly more negative for the above-median values of $\hat{E}_{q}$ $\left(r_{s}=-0.1\right)$ than the below median values of $\hat{E}_{q}\left(r_{\mathrm{s}}\right.$ for below median $E_{q}=0.05, P=.0037$, calculated by bootstrapping the difference in $r_{\mathrm{s}}$ by taking 1,000 random subsamples of $n=100$ from each $\hat{E}_{q}$ distribution).

Finally, we ask whether meta-analyses to obtain distributions of estimates of $E$ are indeed significantly affected by the combination of curvature in the Arrhenius plot and variation in choice of range-location (fig. 3). In this regard, note that range itself does not have a strong effect on $E$ estimates (fig. 2). Specifically, the combined effects of curvature and choice of range-location should result in higher as well as more variable estimates of $E$, as predicted by equations (10) and (11) and illustrated in figure 3. For this, we separately plotted and quantified the distributions of $E$ from thermal responses that had downward (negative) curvature in the Arrhenius plot ( $\hat{E}_{q}$; from $54.4 \%$ of the responses) and those that did not ( $E ; 45.6 \%$ of the responses). The results (table 1 ; fig. 4) show that measures of central tendency (mean, median) and higher moments (variance, skewness) of empirical $E$ distributions are indeed higher. That is, when the $\hat{E}$ values from thermal responses without negative (downward) curvature are compared with those from BA-like thermal responses (from curved Arrhenius plots), the $\hat{E}$ values from BA-like responses have a significantly higher mean, median, variance, and skewness (table 1; fig. 4). These results remain qualitatively the same even if we compare $E$ estimates from only the significantly downward-curved responses $(17.8 \%$ of the responses) with those that were not. Furthermore, the same biases are evident even within particular taxonomic groups or trait types (table 1; fig. C1; figs. A1, B1, C1 available online). Thus, curvature in the Arrhenius plot, combined with variation in choice of range location of experimental temperatures within the PTR, can and do strongly affect properties of estimated $E$ distributions.

\section{Discussion}

We have demonstrated that variation in choice of temperature range-location $(\bar{T})$ and range $\left(T_{\text {range }}\right)$ in experiments

Table 1: Differences in properties of distributions of activation energy estimates from thermal responses with $\left(\hat{E}_{q}\right)$ or without $(\hat{E})$ negative curvature in the Arrhenius plot

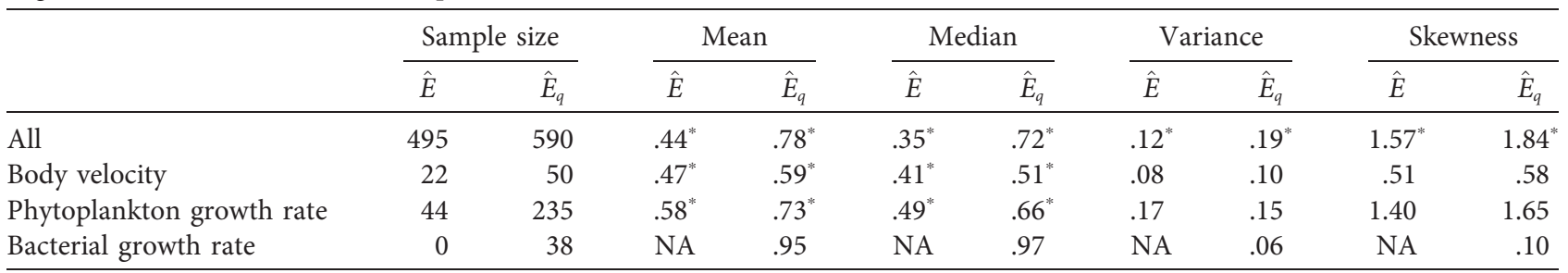

Note: Pairs of properties with significant differences at $P=.01$ are flagged with an asterisk. Each $P$ value was obtained by calculating how many times the observed difference in a property of the activation energy distribution (mean, median, variance, or skewness) was exceeded by the difference between 10,000 bootstrapped differences. The size of each random subsample for the bootstrapping was set to be the smaller of the two data subsets (e.g., resample size is 495 for All). The number of thermal responses for bacterial growth rates was insufficient to test for differences between $\hat{E}$ and $\hat{E}_{q}$ distributions. Also see figures 4 and C1. NA $=$ not applicable. 


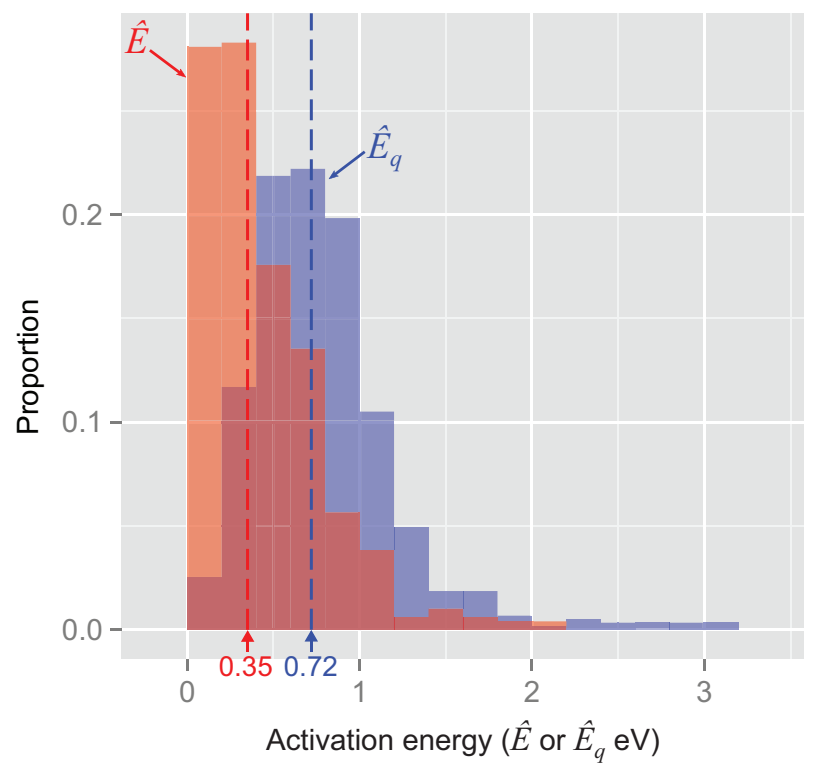

Figure 4: Differences in distributions of activation energy estimates from Boltzmann-Arrhenius-like thermal responses $\left(\hat{E}_{q}\right.$, estimated from downward-curved Arrhenius plots) or those without downward curvature $(\hat{E})$ in the Arrhenius plot (blue and red distributions, respectively). The $\hat{E}_{q}$ values (blue distribution) have a significantly higher mean, median (marked by arrows), variance, and skewness (table 1) These results show that curvature in the Arrhenius plot, combined with variation in the range location of experimental or model-fitting temperatures, can significantly change properties of the estimated $E$ distribution.

and during model fitting are likely to be important contributors to the variation in activation energies of metabolic traits reported by recent studies (Irlich et al. 2009; Dell et al. 2011; Englund et al. 2011; Nilsson-Örtman et al. 2012). The variation can arise, to different degrees, through effects of temperature range, range location, and range spread (eqq. [4][6]) on precision and accuracy of $E$ estimates.

In particular, when there is curvature in the Arrhenius plot, range-location becomes a particularly important source of variation and bias (figs. 3, 4, C1; table 1), with the result that BA-like thermal responses showing downward curvature in the Arrhenius plot yield distributions of thermal sensitivity with significantly higher mean, median, variance, and skewness. Thus, in particular, the right-skewness in activation energies that has been reported in recent studies (Dell et al. 2011; Nilsson-Örtman et al. 2012), may partly be explained by variation in the sampling of range location across studies. That is, as equation (10) (illustrated in fig. 3) shows, a choice of experimental range locations biased toward suboptimal temperatures (toward $T_{\min }$ ) will amplify the right tail of the $\hat{E}_{q}$ distribution if the Arrhenius plot is curved. In this context, it is important to note that curvature in the Arrhenius plot is inevitable if the upper limit of the PTR is defined to be exactly at $T_{\mathrm{pk}}$ (fig. 1 ). This is because the rate of increase in performance with temperature necessarily slows down as $T_{\mathrm{pk}}$ approaches. A solution to reduce this effect is to fit a full unimodal model to data using nonlinear regression (Schoolfield et al. 1981; Ratkowsky et al. 1983; Dell et al. 2011; Barneche et al. 2014), possibly combined with Bayesian inference of the error distribution of the estimated parameter (Corkrey et al. 2012, 2014; Barneche et al. 2014; Johnson et al. 2015). However, due to logistical reasons, sampling full, unimodal thermal responses is often not possible. For example, in our combined data set, only $33.9 \%$ of the thermal responses were unimodal. Without these minimal criteria, nonlinear regression-fitting algorithms either do not converge or the fits are unreliable (Seber and Wild 2003).

And even if data do allow fitting of a unimodal model, insufficient sampling of temperature range and range location will still yield imprecise and biased estimates of thermal sensitivity for the reasons outlined above. As an explicit example, consider the unimodal model

$$
B=B_{0} e^{-E_{a} / k T} /\left(1+e^{-E_{1} / k T}+e^{-E_{h} / k T}\right),
$$

where $E_{\mathrm{a}}$ is activation energy, $E_{\mathrm{l}}$ is low-temperature enzyme deactivation energy, and $E_{\mathrm{h}}$ is high-temperature enzyme deactivation energy (Sharpe and DeMichele 1977; Schoolfield et al. 1981). This model necessarily yields a curved Arrhenius plot within the PTR; in figure $1 C, E_{\max }$ would be analogous to $E_{1}$, and $E_{\min }$ would be analogous to $E_{\mathrm{a}}$. Then, if the PTR is not sufficiently sampled at low temperatures (relevant to $E_{1}$ ), for a trait that actually showed low-temperature inactivation, fitting this unimodal model would yield both inaccurate and imprecise estimates of $E_{1}$ and possibly inflated estimates of $E_{\mathrm{a}}$, lying between $E_{\mathrm{a}}$ and $E_{1}$.

Therefore, poor coverage of the PTR, combined with curvature in the Arrhenius plot, needs to be accounted for in meta-analyses that explore whether biochemical reaction kinetics are sufficient to predict the effects of temperature on biological traits. Indeed, the fact that experimental temperature choices and subsequent model fitting can generate so much variation in $E$ estimates adds an important perspective to the ongoing debate on the adequacy of the BA model and biochemical reaction kinetics for modeling thermal dependence of biological traits (Clarke and Fraser 2004; Gillooly et al. 2006; Irlich et al. 2009; Knies and Kingsolver 2010; Dell et al. 2011; Englund et al. 2011). To illustrate the magnitude of the variation and its implications, consider a curved Arrhenius plot with a curvature coefficient $q_{2}=-0.125$ (the average of coefficients in real data; also see Englund et al. 2011). Interestingly, this curvature coefficient would yield $\hat{E}_{q}=0.65$, a value often quoted in the context of the metabolic theory of ecology, if the entire width of the PTR were 
covered by every experiment. However, if only subsets of the PTR were covered, such that $\bar{T}$ varies substantially across experiments (e.g., the $\mathrm{U}$ and $\mathrm{L}$ range-locations in fig. 1C), $\hat{E}_{q, \text { range }}=1.02$ (eq. [11]). $\hat{E}_{q}$ can take values in the range $0.14-1.16$, depending upon where range-location $\bar{T}$ lies with a theoretical PTR of $0^{\circ}-40^{\circ} \mathrm{C}$. This is remarkably similar to the range expected from the variation in known activation energies of key metabolic reactions $(\sim 0.2$ to $1.2 \mathrm{eV}$; Johnson et al. 1974; Schoolfield et al. 1981; Raven and Geider 1988; Gillooly et al. 2001). In fact, this close correspondence might suggest that thermal responses with a separate lowtemperature activation energy within the PTR (separated from the second activation energy by an Arrhenius breakpoint temperature) are not uncommon across taxa and traits. In such cases, assuming that a single $E$ adequately captures the thermal sensitivity of the organism would be incorrect, and a redefinition of one end of the PTR to be the breakpoint temperature may be necessary (e.g., Muñoz et al. 2014).

Thus, given data limitations, variation in temperature range and range-location in experiments and model fitting will continue to result in inflated variation in activation energies in meta-analyses. In such situations, our results provide guidelines for minimizing artifacts arising from the choice of temperature range, range-spread, and range-location in future experimental studies, as well as meta-analyses, and assessing when systematic deviations from the BA model are too extreme to justify its use in ecological and evolutionary models: (i) Sample from a few temperatures near the anticipated upper limit of the PTR for the study organism(s), so that $T_{\mathrm{pk}}$ can be better identified and thus the true width of the PTR determined. Note that $T_{\mathrm{pk}}$ is expected to be close to the average ambient temperature of the organism's environment. (ii) From $T_{\mathrm{pk}}$, sample toward lower temperatures over a range of at least $10^{\circ} \mathrm{C}$, while keeping in mind that, even for the lowest feasible $n(n=4)$, an order of magnitude increase in range-spread can triple precision (eq. [8]; fig. 2). (iii) If feasible, sample sufficiently (at least three distinct temperatures) above $T_{\mathrm{pk}}$ to sufficiently characterize the full unimodal curve. (iv) Choose number of distinct temperature treatments, keeping in mind that an order of magnitude increase in treatments $n$ can double precision (eq. [8]; e.g., compare $n=4$ and $n=40$ in fig. 2). (v) Test for curvature in the Arrhenius plot using a preliminary sample and measure curvature sensitivity (eqq. [10] and [11]), and increase range-spread if curvature sensitivity is high. Note also that curvature sensitivity will be minimized when range is centered on the middle of the PTR. (vi) Calculate post hoc corrections to $E$ if curvature in the Arrhenius plot is detected and the PTR has been insufficiently sampled (eq. [10]). These guidelines will yield more accurate measurements of the distributions of activation energies, crucial for reliable predictions of the effects of climatic temperature change on biological systems. Although our main results about the sources of artifact-driven variation in activation energies apply generally across traits and organisms, detecting taxon- or trait-specific patterns and developing corresponding corrections would be an important avenue for future research to develop more accurate, taxonspecific estimates of variation in thermal sensitivity (e.g., table 1; fig. C1).

The wide range of $E$ possible from experimental and model-fitting artifacts also has potentially crucial implications for developing predictions for effects of climatic temperature on populations, species interactions, communities, and ecosystems (Dossena et al. 2012; Yvon-Durocher et al. 2012; Pawar et al. 2015). In this context, note that even the less variable (and less biased) $\hat{E}$ distribution shows substantial variation in absolute terms, with a central tendency different from 0.6 or $0.65 \mathrm{eV}$ (figs. 4, C1; table 1). Recent work (Irlich et al. 2009; Knies and Kingsolver 2010; Dell et al. 2011) has emphasized that variation in thermal sensitivity is critical for understanding temperature effects on population, community, and ecosystem dynamics (Dell et al. 2014; Pawar et al. 2015). For example, in contrast to a majority of past research that has assumed that the thermal sensitivity of all species in communities and ecosystems are identical, Dell et al. (2014) have recently shown that differences in thermal sensitivity of interacting species can strongly affect consumerresource coexistence and population dynamics (see also Vasseur and McCann 2005; Grigaltchik et al. 2012). Even within populations, differences in thermal sensitivity between traits of the same species (e.g., consumption vs. metabolic rate; Pawar et al. 2012, 2015) can have important consequences for the energy budgets of organisms (Lemoine and Burkepile 2012; Iles 2014). Similarly, differences in the thermal sensitivity of consumers and resources may also have strong effects on coevolutionary dynamics (Pawar et al. 2015) and differences between pathogens, vectors, and hosts on disease dynamics (Mordecai et al. 2013; Johnson et al. 2015). Recent work also shows that differences in thermal sensitivity between respiration, photosynthesis, and methane production are significant and likely to be of global consequence in the face of a warming climate (Yvon-Durocher et al. 2012, 2014). In all these contexts, thermal acclimation-rapid, nonevolutionary changes in thermal response curves - are likely to play a key role by ameliorating differences between species (Angilletta 2009; Way and Yamori 2014; Pawar et al. 2015). But here again, to develop a general understanding of acclimation rates in thermal sensitivity across organisms, accurate estimates of $\hat{E}$ are crucial (Grigaltchik et al. 2012; Seebacher et al. 2014) to uncover the mechanistic basis (e.g. biochemical constraints vs. behavioral thermoregulation) of acclimation responses.

Accurately quantifying variation in thermal sensitivity between and within species is a new and important challenge in the path of ongoing efforts to predict the effects 
of climate change on the dynamics of biological systems ranging from individuals to whole ecosystems. Inaccuracies in estimates of activation energies can further complicate the already challenging task of uncovering the mechanistic basis of thermal responses of biological traits and, ultimately, understanding and predicting the biological impacts of historical and ongoing climatic fluctuations, as well as global warming.

\section{Acknowledgments}

We thank M. Kearney, E. Landaw, and two anonymous reviewers for helpful comments. S.P. was supported by University of California at Los Angeles (UCLA) Biomathematics start-up funds, National Science Foundation (NSF) Division of Environmental Biology (DEB) award 1021010, and the Grand Challenges in Ecosystems and Environment Initiative at Silwood Park, Imperial College. A.I.D. and V.M.S were supported by UCLA Biomathematics start-up funds and NSF DEB award 1021010. J.L.K. was supported by National Institutes of Health grant F32GM861052.

\section{Literature Cited}

Allen, A. P., J. H. Brown, and J. F. Gillooly. 2002. Global biodiversity, biochemical kinetics, and the energetic-equivalence rule. Science 297:1545-1548.

Angilletta, M. 2009. Thermal adaptation: a theoretical and empirical synthesis. Oxford University Press, Oxford.

Barneche, D. R., M. Kulbicki, S. R. Floeter, A. M. Friedlander, J. Maina, and A. P. Allen. 2014. Scaling metabolism from individuals to reef-fish communities at broad spatial scales. B. Worm, ed. Ecology Letters 17:1067-1076.

Brown, J. H., J. F. Gillooly, A. P. Allen, V. M. Savage, and G. B. West. 2004. Toward a metabolic theory of ecology. Ecology 85:17711789 .

Clarke, A. 2004. Is there a universal temperature dependence of metabolism? Functional Ecology 18:252-256.

Clarke, A., and K. P. P. Fraser. 2004. Why does metabolism scale with temperature? Functional Ecology 18:243-251.

Corkrey, R., T. A. McMeekin, J. P. Bowman, D. A. Ratkowsky, J. Olley, and T. Ross. 2014. Protein thermodynamics can be predicted directly from biological growth rates. PLoS ONE 9:e96100.

Corkrey, R., J. Olley, D. Ratkowsky, T. McMeekin, and T. Ross. 2012. Universality of thermodynamic constants governing biological growth rates. PLos ONE 7:e32003.

Dell, A. I., S. Pawar, and V. M. Savage. 2011. Systematic variation in the temperature dependence of physiological and ecological traits. Proceedings of the National Academy of Sciences of the USA 108: 10591-10596.

- 2013. The thermal dependence of biological traits. Ecology 94:1205.

- 2014. Temperature dependence of trophic interactions are driven by asymmetry of species responses and foraging strategy. Journal of Animal Ecology 83:70-84.

Del Rio, C. M. 2008. Metabolic theory or metabolic models? Trends in Ecology and Evolution 23:256-260.
Deutsch, C. A., J. Tewksbury, R. B. Huey, K. S. Sheldon, C. K. Ghalambor, D. C. Haak, and P. R. Martin. 2008. Impacts of climate warming on terrestrial ectotherms across latitude. Proceedings of the National Academy of Sciences of the USA 105:66686672.

Dillon, M. E., G. Wang, and R. B. Huey. 2010. Global metabolic impacts of recent climate warming. Nature 467:704-706.

Dossena, M., G. Yvon-Durocher, J. Grey, J. M. Montoya, D. M. Perkins, M. Trimmer, and G. Woodward. 2012. Warming alters community size structure and ecosystem functioning. Proceedings of the Royal Society B: Biological Sciences 279:3011-3019.

Englund, G., G. Ohlund, C. L. Hein, and S. Diehl. 2011. Temperature dependence of the functional response. Ecology Letters 14:914-921.

Freitas, V., J. F. M. F. Cardoso, K. Lika, M. A. Peck, J. Campos, S. A. L. M. Kooijman, and H. W. van der Veer. 2010. Temperature tolerance and energetics: a dynamic energy budget-based comparison of North Atlantic marine species. Philosophical Transactions of the Roval Societv B: Biological Sciences 365:3553-3565.

Gillooly, J., A. Allen, V. M. Savage, E. L. Charnov, G. B. West, and J. H. Brown. 2006. Response to Clarke and Fraser: effects of temperature on metabolic rate. Functional Ecology 20:400-404.

Gillooly, J. F., J. H. Brown, G. B. West, V. M. Savage, and E. L. Charnov. 2001. Effects of size and temperature on metabolic rate. Science 293:2248-2251.

Grigaltchik, V. S., A. J. W. Ward, and F. Seebacher. 2012. Thermal acclimation of interactions: differential responses to temperature change alter predator-prey relationship. Proceedings of the Roval Society B: Biological Sciences 279:4058-4064.

Hobbs, J. K., W. Jiao, A. D. Easter, E. J. Parker, L. A. Schipper, and V. L. Arcus. 2013. Change in heat capacity for enzyme catalysis determines temperature dependence of enzyme catalyzed rates. ACS Chemical Biology 8:2388-2393.

Huey, R. B., M. R. Kearney, A. Krockenberger, J. A. M. Holtum, M. Jess, and S. E. Williams. 2012. Predicting organismal vulnerability to climate warming: roles of behaviour, physiology and adaptation. Philosophical Transactions of the Roval Societv B: Biological Sciences 367:1665-1679.

Huey, R. B., and J. G. Kingsolver. 2011. Variation in universal temperature dependence of biological rates. Proceedings of the National Academy of Sciences of the USA 108:10377-10378.

Iles, A. C. 2014. Towards predicting community level effects of climate: relative temperature scaling of metabolic and ingestion rates. Ecology 95:2657-2668.

Irlich, U. M., J. S. Terblanche, T. M. Blackburn, and S. L. Chown. 2009. Insect rate-temperature relationships: environmental variation and the metabolic theory of ecology. American Naturalist 174: 819-835.

Johnson, F., H. Eyring, and B. Stover. 1974. The theory of rate processes in biology and medicine. Wiley, New York.

Johnson, L., T. Ben-Horin, K. D. Lafferty, A. McNally, E. Mordecai, K. P. Paaijmans, S. Pawar, et al. 2015. Understanding uncertainty in temperature effects on vector-borne disease: a Bayesian approach. Ecology 96:203-213.

Kearney, M., and W. Porter. 2009. Mechanistic niche modelling: combining physiological and spatial data to predict species' ranges. Ecology Letters 12:334-350.

Kingsolver, J. G., H. Arthur Woods, L. B. Buckley, K. A. Potter, H. J. MacLean, and J. K. Higgins. 2011. Complex life cycles and the responses of insects to climate change. Integrative and Comparative Biology 51:719-732. 
Knies, J. L., and J. G. Kingsolver. 2010. Erroneous Arrhenius: modified Arrhenius model best explains the temperature dependence of ectotherm fitness. American Naturalist 176:227-233.

Kooijman, S. A. L. M. 2000. Dynamic energy and mass budgets in biological svstems. Cambridge University Press, Cambridge.

Kruse, J., H. Rennenberg, and M. A. Adams. 2011. Steps towards a mechanistic understanding of respiratory temperature responses. New Phytologist 189:659-677.

Lehmann, E. L., and G. Casella. 1998. Theory of point estimation. Springer texts in statistics. Springer, New York.

Lemoine, N. P., and D. E. Burkepile. 2012. Temperature-induced mismatches between consumption and metabolism reduce consumer fitness. Ecology 93:2483-2489.

Martin, T. L., and R. B. Huey. 2008. Why "suboptimal" is optimal: Jensen's inequality and ectotherm thermal preferences. American Naturalist 171:E102-E118.

McArdle, B. H. 1988. The structural relationship: regression in biology. Canadian Journal of Zoology 66:2329-2339.

McMeekin, T., J. Olley, D. Ratkowsky, R. Corkrey, and T. Ross. 2013. Predictive microbiology theory and application: is it all about rates? Food Control 29:290-299.

Mordecai, E., K. Paaijmans, L. R. Johnson, C. Balzer, T. Ben-Horin, E. DeMoor, A. McNally, et al. 2013. Optimal temperature for malaria transmission is dramatically lower than previously predicted. Ecology Letters 16:22-30.

Muñoz, N. J., A. P. Farrell, J. W. Heath, and B. D. Neff. 2014. Adaptive potential of a Pacific salmon challenged by climate change. Nature Climate Change 5:163-166.

Nilsson-Örtman, V., R. Stoks, M. De Block, H. Johansson, F. Johansson, and B. Enquist. 2012. Latitudinally structured variation in the temperature dependence of damselfly growth rates. Ecology Letters 64-71.

O'Connor, M. I., B. Gilbert, C. J. Brown, M. I. O'Connor, A. E. C. M. Del Rio, and E. J. L. Bronstein. 2011. Theoretical predictions for how temperature affects the dynamics of interacting herbivores and plants. American Naturalist 178:626-638.

Ouedraogo, A., J. Fargues, M. S. Goettel, and C. J. Lomer. 1997. Effect of temperature on vegetative growth among isolates of Metarhizium anisopliae and M. flavoviride. Mycopathologia 137:37-43.

Pawar, S., A. I. Dell, and V. M. Savage. 2012. Dimensionality of consumer search space drives trophic interaction strengths. Nature 486:485-489.

2015. From metabolic constraints on individuals to the ecoevolutionary dynamics of ecosystems. Pages 3-36 in A. Belgrano, G. Woodward, and U. Jacob, eds. Aquatic functional biodiversity: an eco-evolutionary approach. Elsevier, Amsterdam.

Petchey, O. L., U. Brose, and B. C. Rall. 2010. Predicting the effects of temperature on food web connectance. Philosophical Transactions of the Roval Society B: Biological Sciences 365:2081-2091.

Rall, B. C., O. Vucic-Pestic, R. B. Ehnes, M. Emmerson, and U. Brose. 2010. Temperature, predator-prey interaction strength and population stability. Global Change Biology 16:2145-2157.

Ratkowsky, D. A., R. K. Lowry, T. A. McMeekin, A. N. Stokes, and R. E. Chandler. 1983. Model for bacterial culture growth rate throughout the entire biokinetic temperature range. Journal of Bacteriology 154:1222-1226.

Ratkowsky, D. A., J. Olley, and T. Ross. 2005. Unifying temperature effects on the growth rate of bacteria and the stability of globular proteins. Lournal of Theoretical Biology 233:351-362.
Raven, J. A., and R. J. Geider. 1988. Temperature and algal growth New Phvtologist 110:441-461.

Savage, V. M., J. F. Gilloly, J. H. Brown, E. L. Charnov, J. F. Gillooly, and G. B. West. 2004. Effects of body size and temperature on population growth. American Naturalist 163:429-441.

Schoolfield, R. M., P. J. Sharpe, and C. E. Magnuson. 1981. Non-linear regression of biological temperature-dependent rate models based on absolute reaction-rate theory. Lournal of Theoretical Biology 88:719-731.

Seber, G. A. F., and C. J. Wild. 2003. Nonlinear regression. Wiley, New York.

Seebacher, F., C. R. White, and C. E. Franklin. 2014. Physiological plasticity increases resilience of ectothermic animals to climate change. Nature Climate Change 5:61-66.

Sharpe, P. J., and D. W. DeMichele. 1977. Reaction kinetics of poikilotherm development. Journal of Theoretical Biology 64:649-670.

Silvius, J. R., and R. N. McElhaney. 1981. Non-linear Arrhenius plots and the analysis of reaction and motional rates in biological membranes. Lournal of Theoretical Biology 88:135-152.

Stegen, J. C., R. Ferriere, and B. J. Enquist. 2012. Evolving ecological networks and the emergence of biodiversity patterns across temperature gradients. Proceedings of the Roval Societv B: Biological Sciences 279:1051-1060.

Stocker, T. F., D. Qin, G.-K. Plattner, M. Tignor, S. K. Allen, J. Boschung, A. Nauels, et al. 2013. Climate change 2013: the physical science basis. Cambridge University Press, New York.

Sunday, J. M., A. E. Bates, M. R. Kearney, R. K. Colwell, N. K. Dulvy, J. T. Longino, and R. B. Huey. 2014. Thermal-safety margins and the necessity of thermoregulatory behavior across latitude and elevation. Proceedings of the National Academv of Sciences of the USA 111:5610-5615.

Thomas, M. K., C. T. Kremer, C. A. Klausmeier, and E. Litchman. 2012. A global pattern of thermal adaptation in marine phytoplankton. Science 338:1085-1088.

Vasseur, D. A., and K. S. McCann. 2005. A mechanistic approach for modeling temperature-dependent consumer-resource dynamics. American Naturalist 166:184-198.

Way, D. A., and W. Yamori. 2014. Thermal acclimation of photosynthesis: on the importance of adjusting our definitions and accounting for thermal acclimation of respiration. Photosynthesis Research 119:89-100.

Wolfe, J., and D. J. Bagnall. 1980. Arrhenius plots: curves or straight lines? Annals of Botany 45:485.

Wolfshaar, K. E. van de, A. M. de Roos, and L. Persson. 2008. Population feedback after successful invasion leads to ecological suicide in seasonal environments. Ecology 89:259-268.

Yvon-Durocher, G., A. P. Allen, D. Bastviken, R. Conrad, C. Gudasz, A. St-Pierre, N. Thanh-Duc, et al. 2014. Methane fluxes show consistent temperature dependence across microbial to ecosystem scales. Nature 507:488-491.

Yvon-Durocher, G., J. M. Caffrey, A. Cescatti, M. Dossena, P. del Giorgio, J. M. Gasol, J. M. Montoya, et al. 2012. Reconciling the temperature dependence of respiration across timescales and ecosystem types. Nature 487:472-476.

Associate Editor: Michael Kearney Editor: Susan Kalisz 


\section{Appendix A from S. Pawar et al., "Real versus Artificial Variation in the Thermal Sensitivity of Biological Traits" \\ (Am. Nat., vol. 187, no. 2, p. E000)}

\section{Deriving the Curvature-Sensitivity of $E$}

Here we derive the sensitivity of activation energy estimates $\hat{E}$, obtained using ordinary least squares (OLS) fitting, to curvature in the Arrhenius plot of the thermal response of a given biological trait. We term such responses, which have a curved Arrhenius plot, as "Boltzmann-Arrhenius-like responses," or "BA-like thermal responses." We begin by reproducing the standard derivation for the OLS estimator for the linear regression model fitted to the Arrhenius plot of a truly BA (no curvature in the Arrhenius plot) response (eq. [3]). First, we define the residual sum of squares,

$$
S(x, y) \equiv \sum_{i=1}^{n}\left[y_{i}-\left(c+\hat{m} x_{i}\right)\right]^{2},
$$

and find the values of $c$ and $m$ that minimize $S(x, y)$ by taking the partial derivatives,

$$
\begin{gathered}
\frac{\partial S(x, y)}{\partial \hat{c}}=\sum_{i=1}^{n} \frac{\partial\left[y_{i}-\left(\hat{c}+\hat{m} x_{i}\right)\right]^{2}}{\partial \hat{c}}=0, \\
\frac{\partial S(x, y)}{\partial \hat{m}}=\sum_{i=1}^{n} \frac{\partial\left[y_{i}-\left(\hat{c}+\hat{m} x_{i}\right)\right]^{2}}{\partial \hat{m}}=0,
\end{gathered}
$$

with $\hat{c}$ and $\hat{m}$ indicating that these are parameter estimates. That is, we need to solve the following equations for these two parameter estimates,

$$
\begin{aligned}
& -2 \sum_{i=1}^{n}\left[y_{i}-\hat{c}-\hat{m} x_{i}\right]=-2\left(-n \hat{c}+\sum_{i=1}^{n} y_{i}-\hat{m} \sum_{i=1}^{n} y_{i}\right)=0, \\
& 2 \sum_{i=1}^{n}\left[x_{i}\left(y_{i}-c-\hat{m} x_{i}\right)\right]=2\left(\sum_{i=1}^{n} x_{i} y_{i}-c \sum_{i=1}^{n} x_{i}-\hat{m} \sum_{i=1}^{n} x_{i}^{2}\right)=0 .
\end{aligned}
$$

These simplify to the following simultaneous equations:

$$
\begin{gathered}
-n c+\sum_{i=1}^{n} y_{i}+\hat{m} \sum_{i=1}^{n} x_{i}=0, \\
\sum_{i=1}^{n} x_{i} y_{i}-c \sum_{i=1}^{n} x_{i}+\hat{m} \sum_{i=1}^{n} x_{i}^{2}=0 .
\end{gathered}
$$

From the first of these two equations, we have

$$
\frac{1}{n}\left(\sum_{i=1}^{n} y_{i}+\hat{m} \sum_{i=1}^{n} x_{i}\right)=\bar{y}+\hat{m} \bar{x} .
$$

Substituting this into the second equation gives

$$
\sum_{i=1}^{n} x_{i} y_{i}-\bar{y} \sum_{i=1}^{n} x_{i}-\hat{m} \bar{x} \sum_{i=1}^{n} x_{i}+\hat{m} \sum_{i=1}^{n} x_{i}^{2}=0 .
$$

That is,

$$
\hat{m}=\frac{\sum_{i=1}^{n} x_{i} y_{i}-\bar{y} \sum_{i=1}^{n} x_{i}}{\bar{x} \sum_{i=1}^{n} x_{i}-\sum_{i=1}^{n} x_{i}^{2}} .
$$

Rearranging the right hand side of this equation gives the standard OLS estimator (eq. [7]). Now, consider what happens if the Arrhenius plot is actually curved (with measurement errors), such that 


$$
y_{i}=q_{0}+q_{1} x_{i}+q_{2} x_{i}^{2}+\varepsilon_{i}, i=1, \ldots, n,
$$

(eq. [9] in the main text). Each such curve has one unique straight line that satisfies the OLS fitting problem. The slope coefficient $(m)$ of this line's equation is an estimate of $E_{q}$. We use the subscript $q$, because curvature in the Arrhenius plot is inconsistent with the BA model per se, and therefore $\hat{E}_{q}$ is not an activation energy in the strictest sense but still represents an effective activation energy. To find the estimator for $m$, we substitute equation (A3) into equation (A2), ignoring measurement errors $\varepsilon_{\mathrm{i}}$ (i.e., assuming the Arrhenius plot is perfectly curved), which after multiplying both numerator and denominator with $n$ and rearranging gives

$$
\hat{m}=\frac{n \sum_{i=1}^{n} x_{i}\left(q_{0}+q_{1} x_{i}+q_{2} x_{i}^{2}\right)-\sum_{i=1}^{n} q_{0}+q_{1} x_{i}+q_{2} x_{i}^{2} \sum_{i=1}^{n} x_{i}}{\left(\sum_{i=1}^{n} x_{i}\right)^{2}-n \sum_{i=1}^{n} x_{i}^{2}} .
$$

This can be reexpressed as

$$
\hat{m}=q_{1}+q_{2} \bar{x}\left(\frac{n\left(n \sum_{i=1}^{n} x_{i}^{3}-\sum_{i=1}^{n} x_{i} \sum_{i=1}^{n} x_{i}^{2}\right)}{\left(\sum_{i=1}^{n} x_{i}\right)^{3}-n \sum_{i=1}^{n} x_{i} \sum_{i=1}^{n} x_{i}^{2}}\right) .
$$

From the properties of finite arithmetic series, the expression in parentheses reduces to a value of 2. Specifically, substituting the formulae $\sum_{i=1}^{n} x_{i}=n(n+1) / 2, \sum_{i=1}^{n} x_{i}^{2}=n(n+1)(2 n+1) / 6$, and $\sum_{i=1}^{n} x_{i}^{3}=n^{2}(n+1)^{2} / 4$ into equation (A4) and simplifying, we get equation (10):

$$
\hat{m}=-\hat{E}_{q} \approx q_{1}+2 q_{2} \bar{x} .
$$

This is an approximation, because the $x_{i}$ values will not strictly form an arithmetic progression even if experimental temperatures $T_{i}$ are evenly placed, because $x_{i}=1 / k T_{i}$. However, it is an excellent approximation, because within the PTR, the transformation $x_{i}=1 / k T_{i}$ is approximately linear (see the discussion following eq. [6]), which ensures that an arithmetic spacing of $T_{i}$ values produces an approximately arithmetic spacing of $x_{i}$ values. The validity of this assumption and the accuracy of the approximation can be measured by calculating the expression in the parentheses of equation (A4) for real data (in our case, the Biotraits data set). The results can be seen in figure A1; calculating the expression in parentheses in equation (A4) for each of 803 thermal responses in the Biotraits database yields values tightly distributed around the value of 2 .

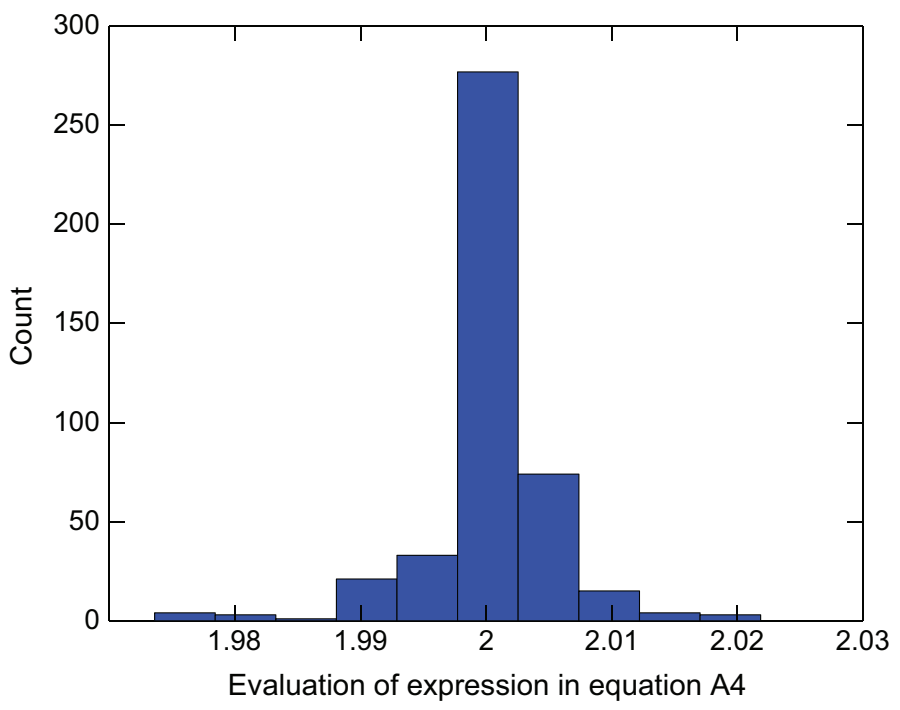

Figure A1: Validation of the assumption that thermal response experiments are performed at temperatures spaced evenly enough to justify the approximation of the sums in equation (A4) that leads to equation (A5), using the temperature measurements from the real data sets we analyze in the main text. 


\section{Appendix B from S. Pawar et al., "Real versus Artificial Variation in the Thermal Sensitivity of Biological Traits"}

\section{(Am. Nat., vol. 187, no. 2, p. E000)}

\section{Validity of the Approximate Mapping between Temperature ( $T$ )-Based and $1 / k T(x)$-Based Measures}
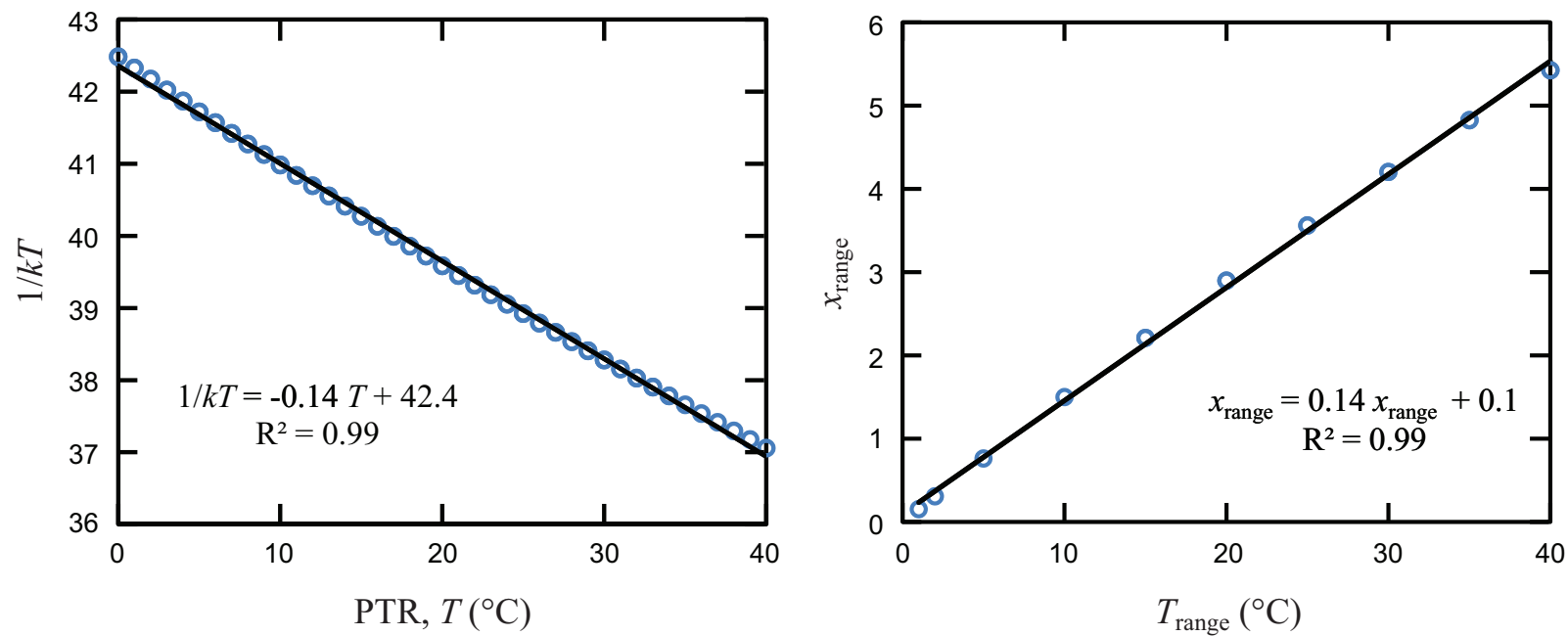

Figure B1: Validation of the relationships in equations (4)-(6), which require linearity of $1 / k T$ over the physiological temperature range (PTR; left). The maximum that $1 / k T$ values (circles) deviate from the linear fit (solid line) is $0.007 \%$. As a result, the relationship between $x_{\text {range }}$ and $T_{\text {range }}(r i g h t)$ is also approximately linear (eq. [4]). The approximate equality and proportionality in equations (5) and (6), respectively, are hence similarly valid. 


\section{Appendix C from S. Pawar et al., "Real versus Artificial Variation in the Thermal Sensitivity of Biological Traits" \\ (Am. Nat., vol. 187, no. 2, p. E000)}

\section{Empirical Results for Certain Traits}

Here we show the results for some specific traits within the Biotraits database (Ratkowsky et al. 2005; Thomas et al. 2012; Dell et al. 2013; fig. C1). We chose the three largest subsets of data that are single-organism traits: bacterial growth rate and body velocity. 

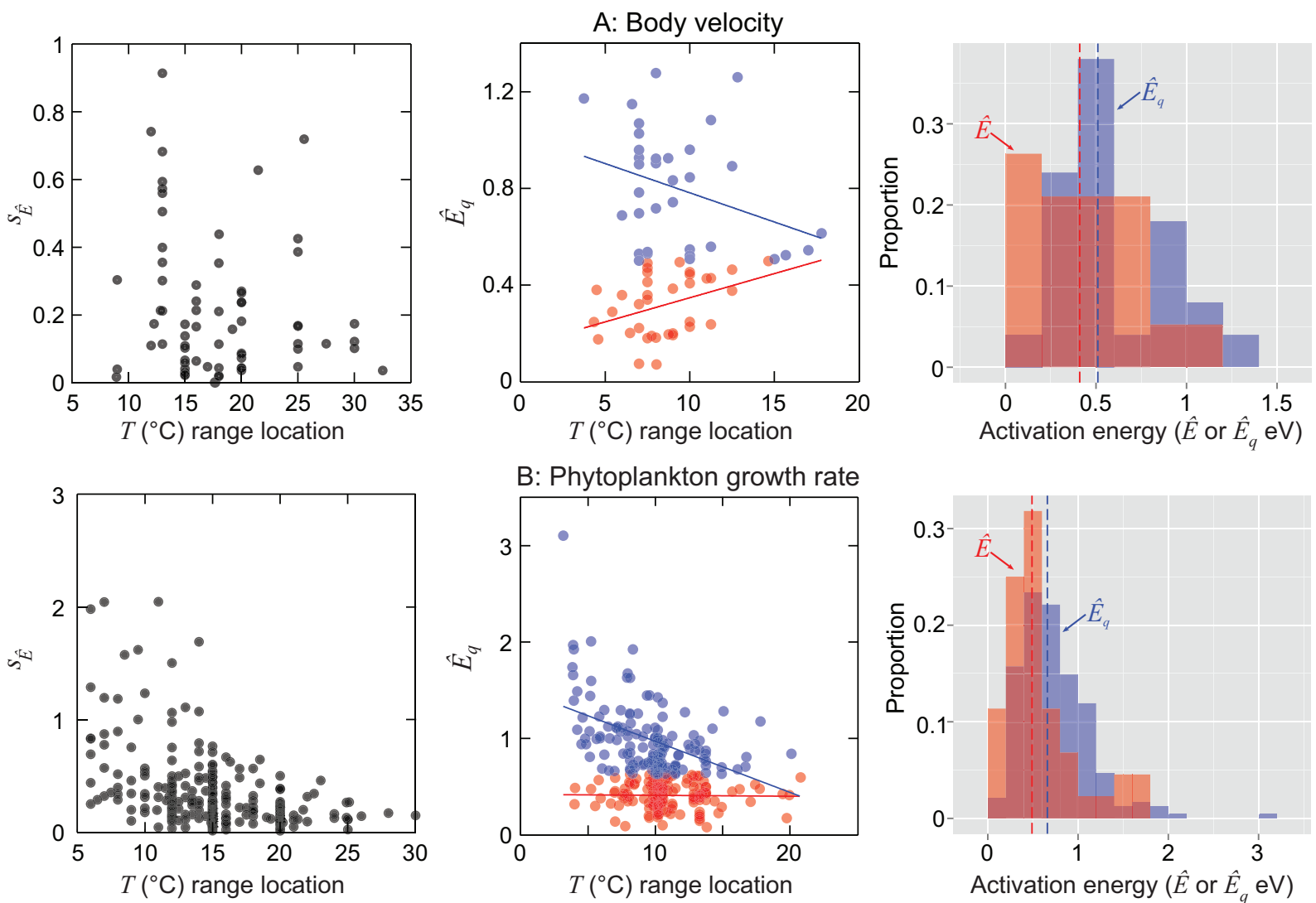

B: Phytoplankton growth rate
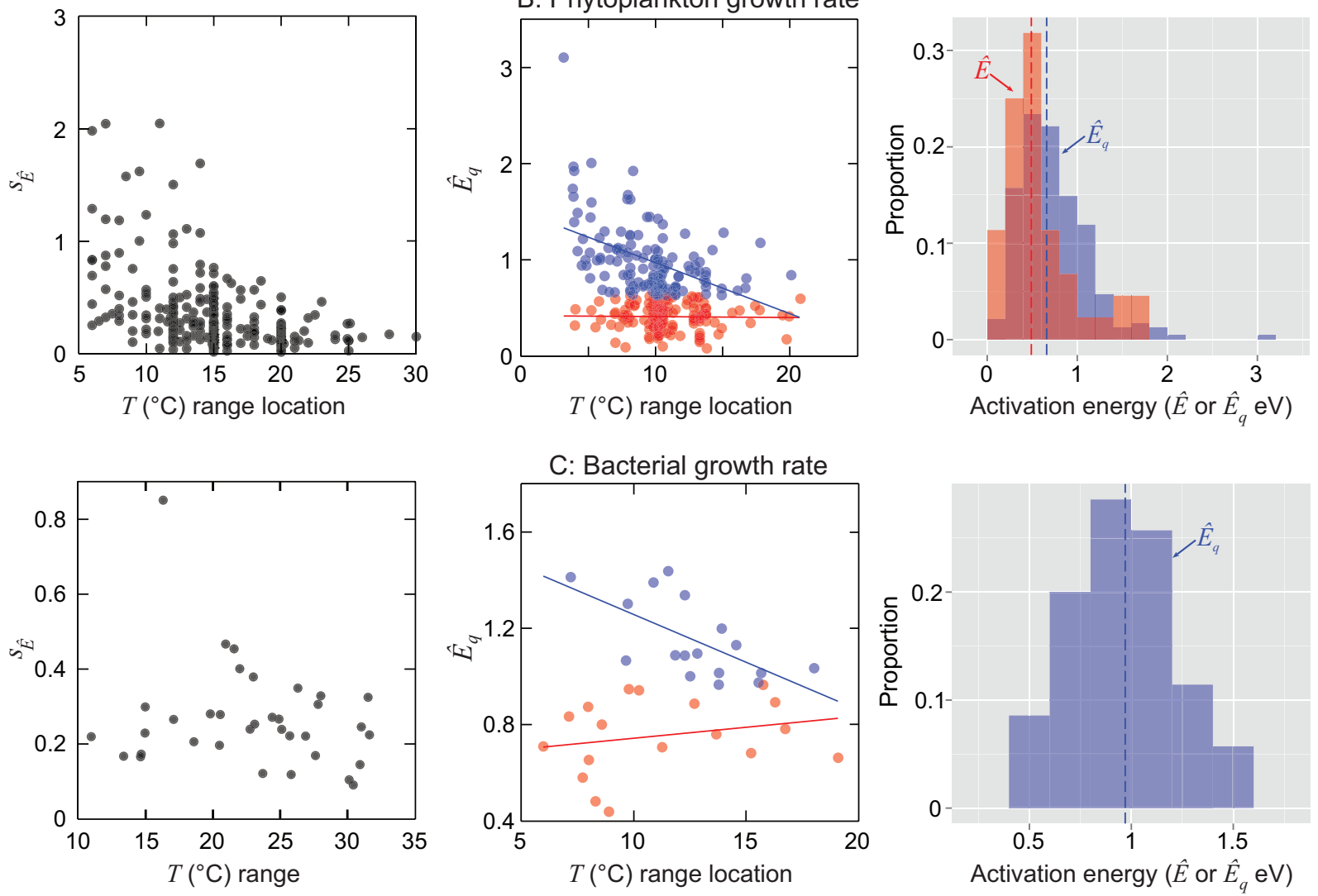

Figure C1: The effect of temperature range on precision (standard error), temperature range-location on $\hat{E}_{q}$, and differences between the $\hat{E}_{q}$ and $\hat{E}$ distributions for three subsets of the full data set. Compare with figure $2 B$ (inset), figure 3 , and figure 4 , respectively. Note that the hyperbolic decrease in standard deviation here is much weaker than those seen in figure $2 B$ (inset), because most of the temperature ranges are $\geq 10^{\circ} \mathrm{C}$. There is no distribution (no red histogram) for $E$ in the bacterial data set $(C)$, because all the thermal responses showed significant downward curvature. 